\title{
Manejo anestésico en la enfermedad de Steinert
}

\section{Anesthetic management in Steinert's disease}

\section{Dra. Andrea Fernanda Quispe-Huamani,* Dr. Renato Francisco Ochoa-Ecos,* Dr. David Bryan Jiménez-Hernández*}

Citar como: Quispe-Huamani AF, Ochoa-Ecos RF, Jiménez-Hernández DB. Manejo anestésico en la enfermedad de Steinert. Rev Mex Anestesiol. 2021; 44 (4): 314. https://dx.doi.org/10.35366/100879

Estimado Sr. Editor:

$\mathrm{H}$ emos leído recientemente su artículo referente al manejo anestésico en la enfermedad de Steinert ${ }^{(1)}$. Se entiende que la distrofia muscular miotónica tipo I, o también llamada comúnmente enfermedad de Steinert, es una miopatía cuya clínica frecuente es miotomía y atrofia muscular. Además, se añade que es una enfermedad multisistemática que llega a dañar tanto el sistema neurológico, cardiológico, respiratorio, endocrinológico y digestivo, siendo una de las enfermedades con gran cantidad de variabilidad clínica ${ }^{(1,2)}$.

Con respecto al beneficio, encontramos que la anestesia locorregional es muy frecuente en pacientes con enfermedad de Steinert por ser segura y por ser la técnica de elección, también tenemos la valoración preanestésica detallada debido a las manifestaciones extramusculares que pueden llegar a amenazar la vida ${ }^{(3)}$.

Con respecto a las debilidades, observamos que no hay muchos informes de incidencia con la vía aérea difícil, pero debemos obviar incitar en el intraoperatorio, también evitar los bloqueadores neuromusculares despolarizantes que en esta enfermedad están contraindicados como opioides que aumentan la sensibilidad(4).

Por otro lado, se entiende que la anestesia locorregional llega a provocar un bloqueo de manera reversible a la conducción del impulso nervioso, éste va ser ayudado por los anestésicos locales, permitiendo anestesiar partes específicas del cuerpo en las personas ${ }^{(1,5)}$.

Gracias a la utilización de la neuroestimulación y la localización ecográfica ayudaron en el amplio desarrollo de las técnicas de la anestesia locorregional, que pueden ser tanto axiales, con anestesia bilateral y simétrica, y también pueden ser más o menos extensas en la zona inferior del cuerpo, o periféricas, mediante un bloqueo selectivo de uno o más nervios de una determinada extremidad. Por último, nos llega a asegurar que la presencia de una analgesia postoperatoria de calidad mejorará la rehabilitación de nuestro paciente ${ }^{(1,5)}$.

Otro punto a detallar es la parte farmacológica, que no hay tratamiento eficaz para esta enfermedad de Steinert, por lo que genera complicaciones secundarias a nivel muscular. Por ello la anestesia es compleja de relacionar, lo que lleva a una intubación difícil en la mayoría de los casos (de los pocos casos reportados, 95\% de ellos son indicados), medicamentos como propanolol, potasio, succinilcolina y anticolinesterásicos son el tipo de procedimiento quirúrgico permitido ${ }^{(3,5)}$.

\section{REFERENCIAS}

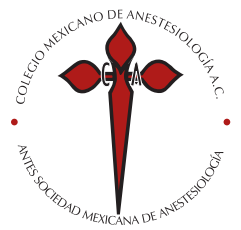

* Estudiante de Medicina, Escuela Profesional de Medicina Humana, Universidad Privada San Juan Bautista, Filial Ica, Perú.

Correspondencia:

Dra. Andrea Fernanda Quispe-Huamani

Carretera Panamericana Sur Km 300, Ica, 11000, Perú.

E-mail: andrea.quispe@upsjb.edu.pe
1. Molero-Díez YB, Sánchez-Hernando VJ, Ruiz-Simón FA, GómezFernández M, Mateos-Arribas MT, García-Lázaro F. Manejo anestésico en la enfermedad de Steinert. A propósito de un caso. Rev Mex Anestesiol. 2021;44:66-69. doi: 10.35366/97780.

2. Rosado-Bartolomé A, Gutiérrez-Gutiérrez G, Prieto-Matos J. Actualización en distrofia miotónica tipo 1 del adulto. Med Fam Semer. 2020;46:355-362.
3. Amores Agulla T, Cantero Domínguez Y, Marrero Quesada J. Anestesia en la enfermedad de Steinert. Rev Cuba Anestesiol Reanim. 2013;12:52-56.

4. Oriol-López SA, Hernández-Bernal CE. Anestesia en la distrofia muscular de Steinert. Rev Mex Anestesiol. 2010;33:160-165.

5. Barmaimon E. Libro anestesia locorregional. Montevideo, Uruguay: Biblioteca Virtual de Salud del S.M.U.; 2017; 5: 4-209. 